\title{
Validation of Antenna Modeling Methodology in IMT-Advanced Channel Model
}

\author{
Jianhua Zhang and Chun Pan \\ Key Laboratory of Universal Wireless Communications, Ministry of Education, Wireless Technology Innovation Institute, \\ Beijing University of Posts and Telecommunications, Beijing 100876, China \\ Correspondence should be addressed to Chun Pan, panchun1987@gmail.com
}

Received 6 July 2012; Revised 28 September 2012; Accepted 7 October 2012

Academic Editor: Guangyi Liu

Copyright ( $\odot 2012$ J. Zhang and C. Pan. This is an open access article distributed under the Creative Commons Attribution License, which permits unrestricted use, distribution, and reproduction in any medium, provided the original work is properly cited.

In this paper, the antenna modeling method in the International Mobile Telecommunications-Advanced (IMT-Advanced) channel model is validated by field channel measurements in the indoor scenario at $2.35 \mathrm{GHz}$. First, the $2 \times 2 \mathrm{MIMO}$ channel impulse responses (CIRs) are recorded with practical antennas as references. Second, the CIRs are reconstructed from the available IMTAdvanced channel model with field patterns of the practical antennas and updated spatial parameters extracted from the similar scenario measurements. Then comparisons between the field CIRs and the reconstructed CIRs are made from coherent bandwidth, eigenvalue dispersion, outage capacity, and ergodic channel capacity. It is found that the reconstructed results closely approximate real results in the coherent bandwidth and correctly describe the statistical characteristics in frequency domain. Compared to the field CIRs, the spatial correlation of the reconstructed CIRs with both types of antenna have a wider range that causes the underestimation of the $5 \%$ channel outage capacity. Due to the negligence of the coupling among the antennas and the near field effect of antenna, this modeling method will have a great impact on the characteristics of radio channels, especially on the spatial characteristics.

\section{Introduction}

Multiple-input multiple-output (MIMO) systems, which deploy spatially separated multiple antenna elements at both ends of the transmission link, have to be considered as one of the most promising approaches for high data rate and more reliable wireless systems without extra bandwidth. It was shown that the potential channel capacity of such MIMO systems grows linearly with antenna pairs in independent identically distributed (i.i.d.) channel model $[1,2]$, but the channel fading correlation affects the capacity by modifying the distributions of the gains of these parallel channels [3]. According to this, the capacity ultimately depends on the propagation channel model used.

Recently, to obtain an accurate channel capacity estimation, many verified channel models [4] were used. In [5], a model based on the Kronecker structure of the channel covariance matrix has been assumed to analyze the channel capacity and was extended to a wideband MIMO channel model in [6]. But in [7], it was confirmed that the Kronecker model cannot describe the multipath structure correctly. For example, the $8 \times 8$ MIMO capacity predicted by the Kronecker model is always below the capacity extracted directly from the filed measurement. Another important wideband MIMO spatial channel model, the International Mobile Telecommunications-Advanced (IMTAdvanced) channel model, is regarded as an appropriate channel model to predict the MIMO channel capacity. This model is a geometry-based stochastic channel model, which adopts the multipath superposition method to generate channel coefficients. By separating multipath channel model into multipath propagation channel model and antenna pattern model, this model not only characterizes the multipath channel parameter but also can be configured with any type of antenna array. So this model is popular to investigate the impact of antenna array configuration on the capacity and the reliability of algorithm. The effects of the user's presence on the performance of a MIMO system in data 
and in voice usage scenarios are investigated in [8], which concludes that the subscriber's usage has much impact on the link performance.

Although this modeling method is convenient to analyze the influences of different antenna configuration on the channel characteristics, based on the double-directional channel model, the multipath parameters are generated independently of a specific antenna, its configuration, and its pattern. Meanwhile, this channel model ignores the nonideal issues on the antennas which will influence the propagation characteristics, such as practical antenna pattern, coupling among the antennas, and human body. To the author's knowledge, there is no investigation to validate the impact of the practical antenna on the spatial channel model of MIMO. So it is not clear if it is accurate enough to model the antenna impact by combining the antenna pattern and multipath propagation channel.

In this paper, the field-measured MIMO channel impulse responses (CIRs) is firstly recorded with practical dipole antenna (DPA) and real terminal planar inverted-F antenna (PIFA) [9] as the reference, in which the channel measurements are conducted in indoor scenario. Second, the CIRs are reconstructed from IMT-Advanced channel model with updated spatial parameters which were measured with omnidirectional antenna array (ODA) and extracted by Space alternating generalized expectation maximization (SAGE) algorithm, and the practical antenna pattern's impact is also incorporated by combining the antenna pattern and multipath propagation channel by matrix manipulation. Then the comparisons between field data and reconstructed data are made from some metrics of MIMO channel [10], such as coherent bandwidth, eigenvalue dispersion, outage capacity, and ergodic channel capacity.

The remainder of the paper is organized as follows. The statistical channel model, channel measurements, and data postprocessing are introduced in Section 2. Section 3 presents the results and the analysis. The analysis of channel characteristics between the measured data and the reconstructed data is presented, for example, the frequency domain in Section 3.1 and the spatial domain in Section 3.2, respectively. The capacity analysis is presented in Section 3.3. Conclusions are drawn in Section 4.

\section{Validation Framework}

In order to validate the accuracy of antenna pattern modeling methodology of IMT-Advanced channel model, the linear time variant MIMO channel response is first defined by the MIMO channel complex matrix $\mathbf{H}(t, \tau)$ of dimension $U \times S$, where $U$ is the number of receiving antennas and $S$ is the number of transmitting antennas. The channel matrix relates the $S \times 1$ complex input vector $\mathbf{x}$ to the $U \times 1$ complex output vector $\mathbf{y}$ during a symbol period after adding the $U \times 1$ white Gaussian noise vector $\mathbf{w}$ as follows:

$$
\mathbf{y}(t)=\sum_{\tau} \mathbf{H}(t, \tau) \mathbf{x}(t)+\mathbf{w}(t)
$$

2.1. Channel Model: IMT-Advanced. In IMT-Advanced channel model [11], the spatial and temporal distributions are parameterized to characterize the MIMO channel. One realization consists of six paths composed of twenty subpaths each. The parameters of all subpaths are calculated for each realization. A temporal channel matrix $\mathbf{H}\left(t_{0}, \tau_{n}\right)$ at $t_{0}$ is created by IMT-Advanced program for each path through the superposition of the subpaths. The entries $h_{u, s}\left(t_{0}, \tau_{n}\right)$ of $\mathbf{H}\left(t_{0}, \tau_{n}\right)$ are

$$
\begin{aligned}
h_{u, s}\left(t_{0}, \tau_{n}\right)= & \sum_{m=1}^{M} \mathbf{F}_{r x, u}^{T}\left(\Omega_{n, m}\right) \mathbf{A}_{n, m} \mathbf{F}_{t x, s}\left(\Phi_{n, m}\right) \\
& \times \exp \left(j d_{u} 2 \pi \lambda_{0}^{-1} \sin \left(\Omega_{n, m}\right)\right) \\
& \times \exp \left(j d_{s} 2 \pi \lambda_{0}^{-1} \sin \left(\Phi_{n, m}\right)\right) \\
& \times \exp \left(j 2 \pi f_{d, n, m} t_{0}\right) .
\end{aligned}
$$

The indexes $u$ and $s$ correspond to the receiving and the transmitting antenna elements, respectively. The $\lambda_{0}$ is the wavelength of the carrier. The number of paths is indexed by $n=\{1, \ldots, 6\}$ and the number of the subpaths is $m=$ $\{1, \ldots, 20\}$. The $\mathbf{F}_{r x, u}$ and $\mathbf{F}_{t x, s}$ are defined as the field pattern of the receiving and the transmitting antenna elements, respectively, in (3) and (4) as follows:

$$
\begin{gathered}
\mathbf{F}_{r x, u}\left(\Omega_{n, m}\right)=\left[\begin{array}{l}
\mathbf{F}_{r x, u, V}\left(\Omega_{n, m}\right) \\
\mathbf{F}_{r x, u, H}\left(\Omega_{n, m}\right)
\end{array}\right], \\
\mathbf{F}_{t x, s}\left(\Phi_{n, m}\right)=\left[\begin{array}{l}
\mathbf{F}_{t x, s, V}\left(\Phi_{n, m}\right) \\
\mathbf{F}_{t x, s, H}\left(\Phi_{n, m}\right)
\end{array}\right] .
\end{gathered}
$$

The $d_{u}$ stands for the distance between the $u$ th receiving antenna element and the first element, the $\mathbf{F}_{r x, u, p}$ is the field pattern of the $u$ th receiving antenna element on the $p$ th polarization, and $p=\{1,2\}$. For transmitting antenna elements, the $d_{s}$ and $\mathbf{F}_{t x, s, p}$ hold the same meanings with the $d_{u}$ and $\mathbf{F}_{r x, u, p}$, respectively. The $\tau_{n}, f_{d, n, m}, \Phi_{n, m}$, and $\Omega_{n, m}$ denote the propagation delay, the doppler shift, the azimuth of departure (AoD), and the azimuth of arrival (AoA) of the $(n, m)$ propagation subpath. And the polarization matrix of the $(n, m)$ subpath $\mathbf{A}_{n, m}$ is defined by

$$
\mathbf{A}_{n, m}=\left[\begin{array}{ll}
\alpha_{n, m, V V} & \alpha_{n, m, V H} \\
\alpha_{n, m, H V} & \alpha_{n, m, H H}
\end{array}\right]
$$

where $\alpha_{n, m, p_{1}, p_{2}}$ denote the polarization gain of the $(n, m)$ subpath from the $p_{1}$ th polarization component to the $p_{2}$ th polarization component.

2.2. Validation Methodology. The rational of the IMTAdvanced channel model is based on the concept of the double-directional mobile radio channel [12]. By separating radio channel model (including antenna) into multipath propagation channel model (including none of the antennas) and antenna pattern model, this modeling method is popular in the researches with multiple antennas at both sides of communication link. During the modeling of CIRs, the IMT-Advanced channel model combines antenna response 
and multipath propagation channel by matrix manipulation to obtain the reconstructed CIRs. The feasibility of this methodology will be focused on in this paper.

In this paper, the CIRs $\mathbf{H}(t, \tau)$ in (1) are obtained, respectively, by field measurement and model reconstruction. The direct data (filed measured) $\mathbf{H}^{\text {meas }}$ is recorded by channel sounder with DPA array and the real terminal PIFA. The indirect data $\mathbf{H}^{\text {model }}$ is reconstructed by IMT-Advanced channel model with antenna pattern of the same antenna array used in direct data recording and extracted propagation channel parameters from measurement with ODA in the same scenarios. In the following subsections, the insight into the channel measurement and channel reconstruction is presented.

So lots of channel measurement campaigns are conducted to obtain the raw data. According to the specific requirements, three types of measurement antenna arrays are needed, including DPAs, PIFA, and ODA. Furthermore, the antenna patterns of DPA and PIFA need to be calibrated as $\mathbf{F}^{\mathrm{DPA}}$ and $\mathbf{F}^{\mathrm{PIFA}}$ before measurement. At the end of data acquisition, four groups of CIRs are obtained. They are $\mathbf{H}_{\mathrm{DPA}}^{\text {meas }}$ collected from field measurement with DPAs, $\mathbf{H}_{\mathrm{PIFA}}^{\text {meas }}$ collected from field measurement with PIFA, $\mathbf{H}_{\mathrm{DPA}}^{\text {model }}$ reconstructed by IMT-Advanced channel model with the antenna pattern of DPAs, and $\mathbf{H}_{\mathrm{PIFA}}^{\text {model }}$ reconstructed by IMT-Advanced channel model with the antenna pattern of PIFA. After acquisition of CIRs, the analysis on the channel characteristics will be performed.

2.3. Measurement Antenna. In the field channel measurement, three types of antenna array are used during the measurement, including DPA, PIFA and ODA. The field CIRs $\mathbf{H}^{\text {meas }}$ are collected by using DPA and PIFA as measurement antenna array. At transmitter (Tx), two DPAs with $4 \lambda_{0}$ antenna spacing are used. Meanwhile two DPAs with $1 / 2 \lambda_{0}$ antenna spacing and a basic PIFA are used at receiver $(\mathrm{Rx})$, respectively. The measured far field radiation patterns $\mathrm{F}^{\mathrm{DPA}}$ and $\mathbf{F}^{\text {PIFA }}$ are given in Figure 1 for each of the MIMO antennas. The patterns are measured in $\mathrm{dBi}$ on the azimuth plane $(x-y)$ for all antennas are given in Figure 2. Both $\mathbf{F}^{\mathrm{DPA}}$ and $\mathbf{F}^{\mathrm{PIFA}}$ are the field radiation patterns of DPA and PIFA near the laptop.

In order to capture the multipath channel parameters in high accuracy, the ODA consisting of 56 cross-polarized elements is used at Tx and Rx. All the elements are mounted in a cylinder, and the spacing between the neighboring elements is half wavelength. Figures 3(a) and 3(b) depict, respectively, the structure and antenna pattern of ODA. The collected data with this antenna array is classified as $\mathbf{H}_{\mathrm{ODA}}$, and this measured data is not used as the direct data $\mathbf{H}^{\text {meas }}$ but used to extract the channel parameters. Then $\mathbf{H}^{\text {model }}$ is obtained by channel reconstruction with these channel parameters and the corresponding antenna pattern.

2.4. Measurement System and Environment. An extensive measurement campaign is performed at center frequency of $2.35 \mathrm{GHz}$ with $50 \mathrm{MHz}$ effective bandwidth, using the Elektrobit PropSound channel sounder. As described in
TABLE 1: Measurement parameters setting.

\begin{tabular}{lc}
\hline Parameters & Setting \\
\hline Carrier frequency & $2.35 \mathrm{GHz}$ \\
Bandwidth & $50 \mathrm{MHz}$ \\
Code length & 255 (DPA, PIFA), 63 (ODA) \\
Tx height & $2.35 \mathrm{~m}$ \\
Rx height & $1 \mathrm{~m}$ \\
Tx antenna number & 2 (DPA), 32 (ODA) \\
Rx antenna number & 2 (DPA or PIFA), 56 (ODA) \\
Max Tx to Rx distance & $30 \mathrm{~m}$ \\
\hline
\end{tabular}

TABLE 2: Angular spread.

\begin{tabular}{lccccc}
\hline & & \multicolumn{2}{c}{$\mathrm{Tx}$} & \multicolumn{2}{c}{$\mathrm{Rx}$} \\
& & $\mu\left({ }^{\circ}\right)$ & $\sigma\left(^{\circ}\right)$ & $\mu\left({ }^{\circ}\right)$ & $\sigma\left(^{\circ}\right)$ \\
\hline \multirow{2}{*}{ Measurement } & LOS & 1.58 & 0.21 & 1.77 & 0.15 \\
& NLOS & 1.61 & 0.23 & 1.91 & 0.19 \\
M. 2135 & LOS & 1.60 & 0.18 & 1.62 & 0.22 \\
& NLOS & 1.62 & 0.25 & 1.77 & 0.16 \\
\hline
\end{tabular}

detail in [13], the sounder works in a time-division multiplexing (TDM) mode. Thus periodic pseudo random binary signals are transmitted between different $\mathrm{Tx}-\mathrm{Rx}$ antenna pairs in sequence. The interval within which all antenna pairs are sounded once is defined as a measurement cycle. Some important characteristics of measurement setup can be found in Table 1. Because of the bandwidth limit of the data bus and the number of antenna element, the code length of measurement system is configured as 63 when using ODAs as test antennas.

The measurement is carried out in the indoor office area. The layout of the open area is illustrated in Figure 4(a). It should be noticed that in open area, the AP indicates the position where the $\mathrm{Tx}$ is fixed at $2.35 \mathrm{~m}$, while the $\mathrm{Rx}$ antenna array is fixed on the desk denoted by red dot.

2.5. Data Postprocessing. In data postprocessing, the CIRs are converted from the raw data firstly. Due to the influence of the band-pass filter in measurement system, and 10\% high frequency component of baseband signal is cut during the postprocessing. So the field CIRs $\mathbf{H}_{\mathrm{DPA}}^{\text {meas }}$ and $\mathbf{H}_{\mathrm{PIFA}}^{\text {meas }}$, are used as the direct CIRs $\mathbf{H}^{\text {meas }}$. And SAGE algorithm is applied to extract channel parameters from the CIRs $\mathbf{H}_{\mathrm{ODA}}$. As an extension of maximum-likelihood (ML) method, the SAGE algorithm provides a joint estimation of the parameter set $\theta_{l}=\left\{\tau_{l}, f_{d, f}, \Phi_{l}, \Omega_{l}, \alpha_{l}\right\}, l=\{1, \ldots, L\}$, with no constrains on the response of antenna array. The definition of all these parameter is the same as Section 2.1. In order to capture all dominant paths that characterizes the propagation environment exactly, totally 120 paths of the strongest power are extracted for each measurement cycle, namely, $L=120$.

The angular spread in all scenarios is listed in Table 2. As shown in the table, the angular spread estimated is consistent with that from IMT-Advanced channel model in indoor scenario. 


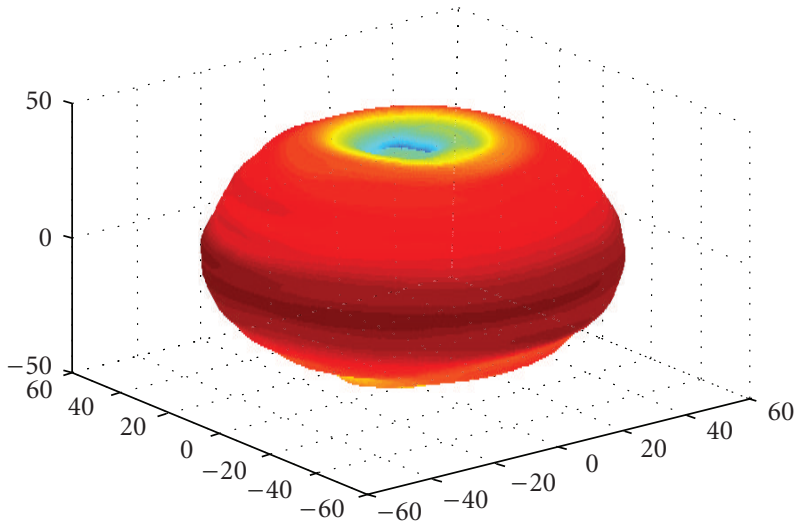

(a)

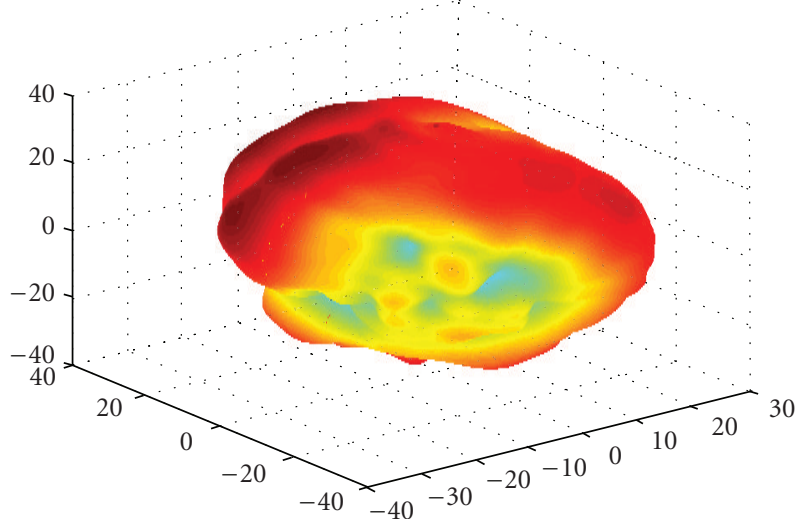

(b)

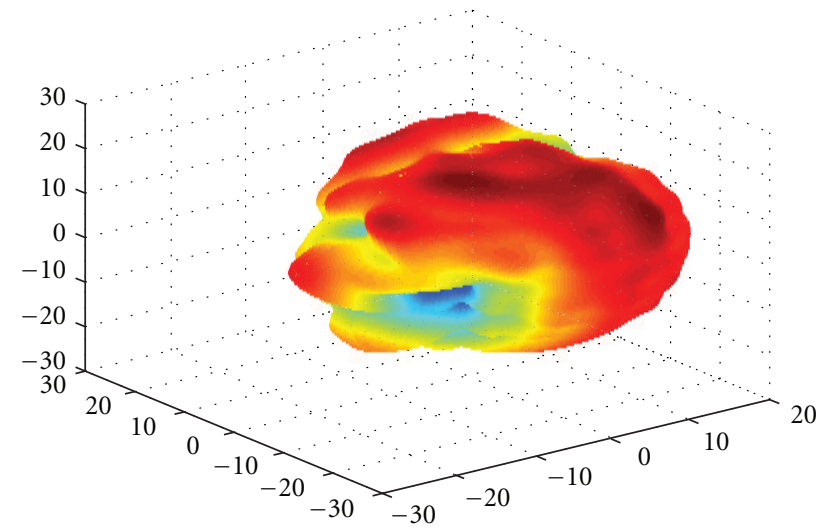

(c)

FIgURE 1: Measured far field radiation patterns for (a) dipole antenna; (b) PIFA antenna port 1; (c) PIFA antenna port 2.

2.6. Channel Reconstruction. Based on IMT-Advanced channel model with the estimated parameter set extracted from CIRs $\mathbf{H}_{\mathrm{ODA}}$, the measured antenna array radiation patterns $\mathbf{F}^{\mathrm{DPA}}$ and $\mathrm{F}^{\mathrm{PIFA}}$ are incorporated into the channel model to obtain the reconstructed CIRs. The channel reconstruction follows the form as below:

$$
\begin{aligned}
h_{u, s}\left(t, \tau_{n}\right)= & {\left[\begin{array}{l}
\mathbf{F}_{r x, u, V}\left(\Omega_{l}\right) \\
\mathbf{F}_{r x, u, H}\left(\Omega_{l}\right)
\end{array}\right]\left[\begin{array}{ll}
\alpha_{l, V V} & \alpha_{l, V H} \\
\alpha_{l, H V} & \alpha_{l, H H}
\end{array}\right]\left[\begin{array}{l}
\mathbf{F}_{t x, s, V}\left(\Phi_{l}\right) \\
\mathbf{F}_{t x, s, H}\left(\Phi_{l}\right)
\end{array}\right] } \\
& \times \exp \left(j d_{u} 2 \pi \lambda_{0}^{-1} \sin \left(\Omega_{l}\right)\right) \\
& \times \exp \left(j d_{s} 2 \pi \lambda_{0}^{-1} \sin \left(\Phi_{l}\right)\right) \times \exp \left(j 2 \pi f_{d, l} t\right) .
\end{aligned}
$$

The antenna pattern $\mathbf{F}_{t x, s}$ at $\mathrm{Tx}$ can be rewritten by $\mathbf{F}^{\mathrm{DPA}}$, while the antenna patterns $\mathbf{F}_{r x, u}$ at $\mathrm{Rx}$ can be rewritten by $\mathbf{F}^{\text {PIFA }}$ or $\mathbf{F}^{\mathrm{DPA}}$. So the reconstructed CIRs $\mathbf{H}^{\text {model }}$ are denoted by $\mathbf{H}_{\mathrm{PIFA}}^{\text {model }}$ and $\mathbf{H}_{\mathrm{DPA}}^{\mathrm{model}}$, respectively. Since there are two antenna elements at both sides of the link, $U=S=2$ in (6).

\section{Analysis and Results}

To analyze the eigenvalue dispersion and capacity of the channel, the corresponding frequency transfer functions $\mathbf{H}(t, f)$ can be obtained by applying the Fourier transform to the CIRs $\mathbf{H}(t, \tau)$. As the spectrum of the transmitted signal reveals a sinc function, signal strength is weak at band edges. Therefore, only $40 \mathrm{MHz}$ in the middle of the band was used for the computation. Assuming that the $\mathbf{H}(j, k)$ is the sample of $\mathbf{H}(t, \tau)$, then

$$
\mathbf{H}(j, k)=\left.\mathbf{H}(t, f)\right|_{t=j \cdot \Delta t, f=k \cdot \Delta f}=\mathbf{H}(j \cdot \Delta t, k \cdot \Delta f),
$$

where $\Delta t$ and $\Delta f$ are the sampling intervals in time domain and frequency domain, respectively. Before the Fourier transform, the noise level estimation should be done to reduce the affect from the additive noise on inherent characteristic of the channel [14]. And the noise level is set to $-71 \mathrm{~dB}$ at spot 4. After denoising, about 6 delay taps are reserved for both the reconstructed data and the field measurement data. The reserved power of real CIRs accounts for $96 \%$ of the signal with noise.

Figure 5 shows the delay domain response of CIRs from field measurement data $\mathbf{H}_{\mathrm{DPA}}^{\mathrm{meas}}$ and the reconstructed data $\mathbf{H}_{\mathrm{DPA}}^{\text {meas }}$ with DPAs. It is obvious that above the noise level the reconstructed data $\mathbf{H}_{\mathrm{DPA}}^{\mathrm{meas}}$ can well fit to the field measurement data $\mathbf{H}_{\mathrm{DPA}}^{\text {meas }}$ in delay domain. 


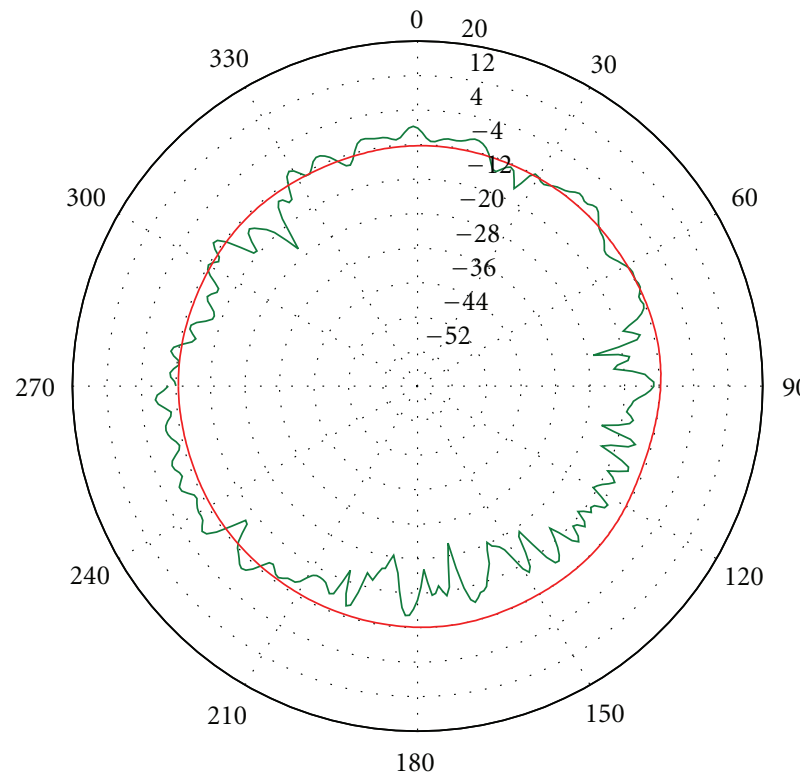

180

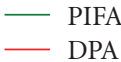

FIGURE 2: Measured radiation patterns (in dBi) on the azimuth plane $(x-y)$ for (a) antenna port 1; (b) antenna port 2.

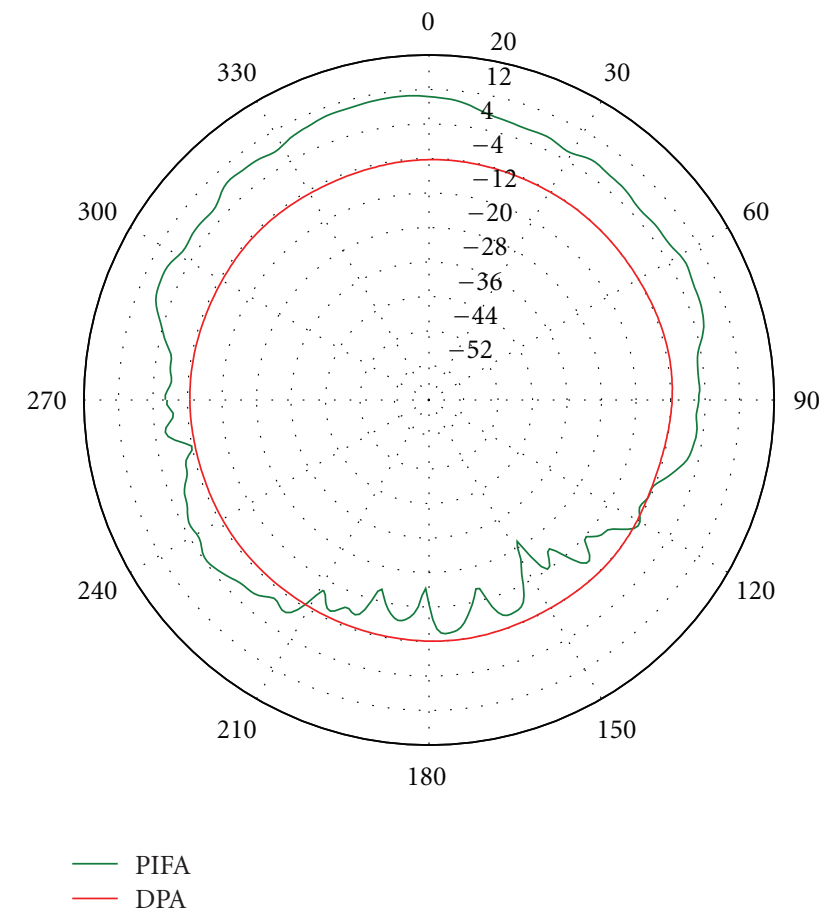

(b) (a)

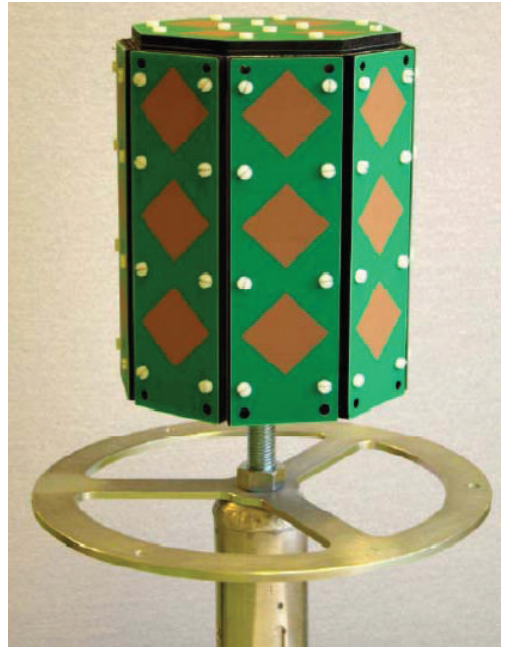

(a)

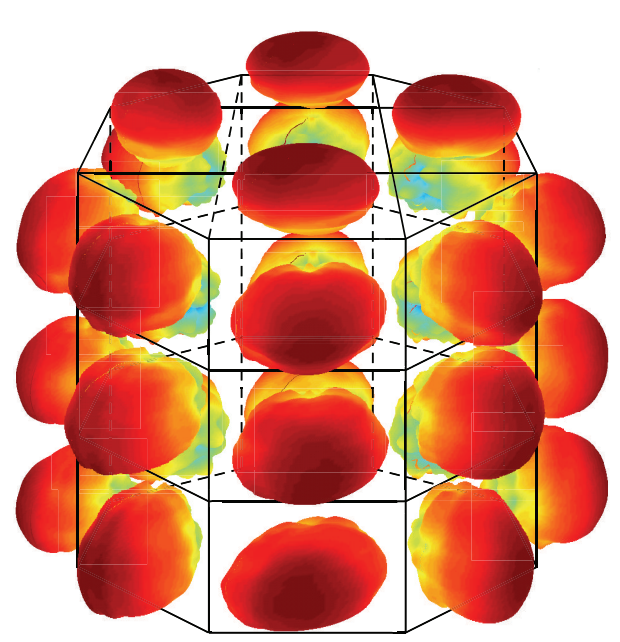

(b)

FIgURE 3: The configurations and antenna pattern of ODA: (a) configurations; (b) antenna pattern.

3.1. Coherent Bandwidth. Based on the CIRs from the measurement and reconstruction, the power delay profile $\beta(\tau)$ can be calculated as

$$
\beta(\tau)=\frac{1}{U S} \sum_{u=1}^{U} \sum_{s=1}^{S}\left|h_{u, s}(\tau)\right|^{2} .
$$

Then a common normalization of the $\beta(\tau)$ is necessary for the probability density function (pdf) $\bar{\beta}(\tau)$. So the delay spread can be calculated as

$$
\sigma_{\tau}=\sqrt{E\left(\tau^{2}\right)-(E(\tau))^{2}},
$$

where $E$ is expectation operator over all channel realizations. The coherent bandwidth is defined as the bandwidth over 


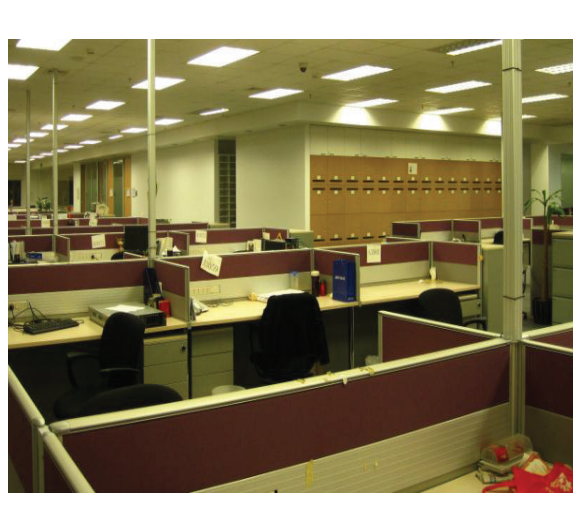

(a)

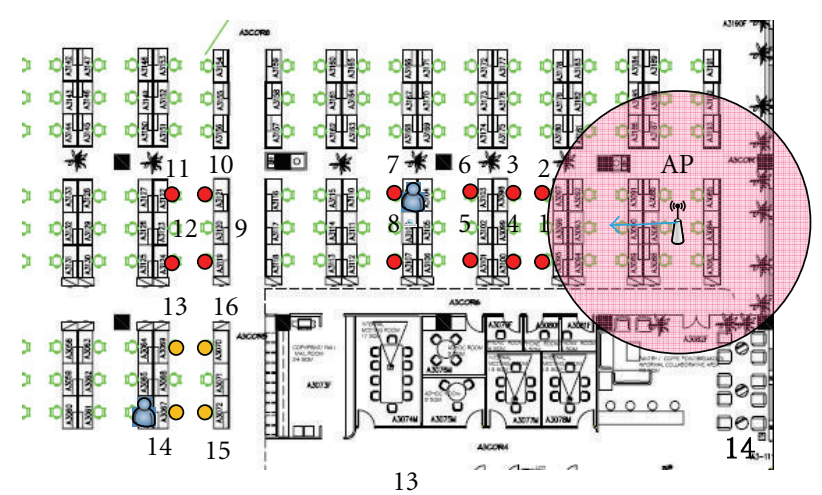

(b)

FIGURE 4: Measurement environment and route plan: (a) measurement environment; (b) route plan.

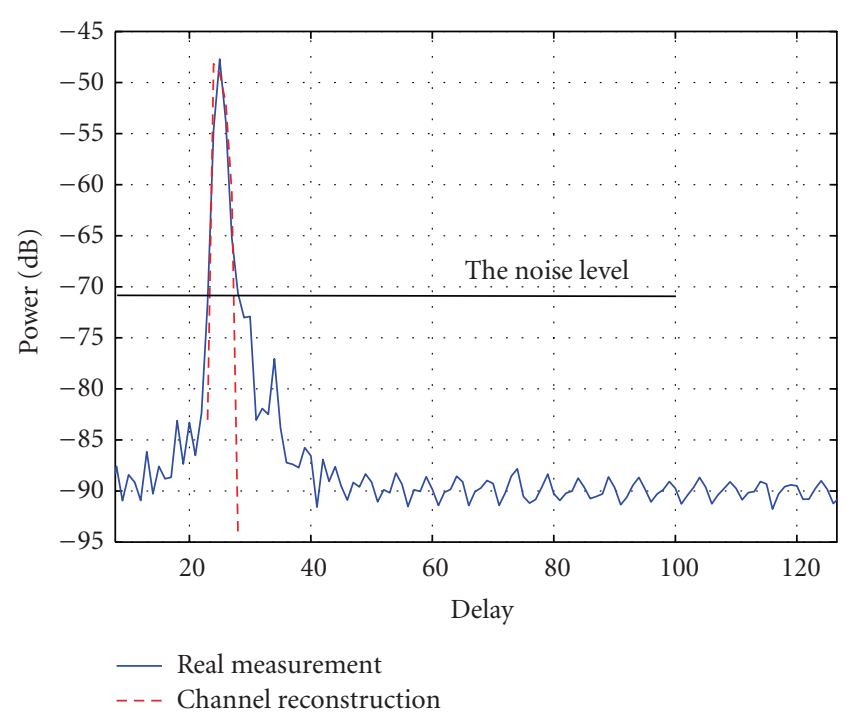

FIGURE 5: The delay domain response (in dB) of CIRs.

which the frequency correlation function is above 0.9 , then the coherent bandwidth can be approximately obtained as

$$
B_{c} \approx \frac{1}{50 \sigma_{\tau}} .
$$

Since $2 \times 2$ antenna configuration is applied in each test case, there are four subchannels between the transmit/receive antenna pairs in each group of CIRs. Table 3 shows the coherent bandwidth of each sub-channel $(\mathrm{CH} 1 \sim \mathrm{CH} 4)$ for the field CIRs $\mathbf{H}^{\text {meas }}$ and the reconstructed CIRs $\mathbf{H}^{\text {model }}$ at spot 4. From the data of the field measurement CIRs $\mathbf{H}^{\text {meas }}$, the difference in coherent bandwidth between DPA and PIFA is not obvious. The small difference in coherent bandwidth of $\mathrm{CH} 2$ and $\mathrm{CH} 4$ can be attributed to the nonideal antenna patterns of PIFA on the azimuth plane as shown in Figure 2(b). For the same reason, the difference of the coherent bandwidth for reconstructed CIRs $\mathbf{H}^{\text {model }}$ is also small.

It is also shown that the coherent bandwidth of the field measurement CIRs $\mathbf{H}_{\mathrm{DPA}}^{\text {meas }}$ and the reconstructed $\mathbf{H}_{\mathrm{DPA}}^{\text {model }}$ are
TABLE 3: The coherent bandwidth (in MHz) of each subchannel in all cases.

\begin{tabular}{llllll}
\hline \multirow{2}{*}{ MEAS } & & $\mathrm{CH} 1$ & $\mathrm{CH} 2$ & $\mathrm{CH} 3$ & $\mathrm{CH} 4$ \\
& DPA & 1.67 & 1.07 & 1.24 & 1.13 \\
\multirow{2}{*}{ MODEL } & PIFA & 1.24 & 0.83 & 1.23 & 0.82 \\
& DPA & 1.26 & 1.27 & 1.37 & 1.28 \\
& PIFA & 1.43 & 1.55 & 1.34 & 1.43 \\
\hline
\end{tabular}

approximated in numerical value. So it is obvious that the reconstruction method based on the IMT-Advanced channel model with DPA will not extend or compress the width of CIRs in delay domain and will not change the statistical characteristics in frequency domain. But the reconstructed CIRs with PIFA will overestimate the coherent bandwidth, especially in $\mathrm{CH} 2$ and $\mathrm{CH} 4$. This is because different from the omnidirectional antenna gain of dipole antenna the nonideal issues on the PIFA pattern compress the width of CIRs and reduce the dispersion in delay domain. Due to the negligence of nonideal antenna pattern modeling in the IMT-Advanced channel model, the reconstructed data will impose a great impact on the system evaluation. In the real environment, the more serious intersymbol interference (ISI) will cause a degradation in the performance of MIMO-OFDM (orthogonal frequency division multiplexing) system.

3.2. Eigenvalue Dispersion. For any MIMO transfer matrix at the $k$ th frequency bin of the $j$ th time realization $\mathbf{H}(j, k)$, the singular value decomposition (SVD) can be obtained as

$$
\begin{aligned}
\mathbf{H}(j, k) & =\mathbf{U}_{\mathrm{SVD}}(j, k) \sum(j, k) \mathbf{V}_{\mathrm{SVD}}^{H}(j, k) \\
& =\sum_{r=1}^{R} \xi_{r}(j, k) u_{r}(j, k) v_{r}^{H}(j, k),
\end{aligned}
$$

where both the $U \times U$ matrix $\mathbf{U}_{\mathrm{SVD}}$ and the $S \times S$ matrix $\mathbf{V}_{\text {SVD }}$ are unitary matrices, and is a $U \times S$ matrix of singular values $\sigma_{i}$ of $\mathbf{H}$. These singular values have the property that for $\xi_{r}$ is the $r$ th largest eigenvalue of $\mathbf{H H}^{H} . \xi_{1}(j, k) \geq$ $\left.\xi_{2}(j, k) \geq \cdots \geq \xi_{R}(j, k)\right), 1 \leq R \leq \min (U, S)$, are the ordered eigenvalues of the $k$ th frequency bin of $j$ th 
TABle 4: The mean value and standard deviation of eigenvalue dispersion.

\begin{tabular}{lccc}
\hline & & Mean & std \\
\hline \multirow{2}{*}{ MEAS } & DPA & 0.65 & 0.12 \\
& PIFA & 0.77 & 0.12 \\
\multirow{2}{*}{ MODEL } & DPA & 0.82 & 0.14 \\
& PIFA & 0.82 & 0.15 \\
\hline
\end{tabular}

time channel realization. $R$ is the rank of channel. The eigenvalue dispersion (ED), which is an important metric of MIMO channel, is commonly used to characterize the relative differences between the powers of eigenvalues. In this paper, we use $S_{\mathrm{ED}}$ as a metric of ED. The $S_{\mathrm{ED}}$ is defined as

$$
S_{\mathrm{ED}}(j, k)=\frac{\left(\prod_{r=1}^{R} \xi_{r}(j, k)\right)^{1 / R}}{(1 / R) \sum_{r=1}^{R} \xi_{r}(j, k)}
$$

which is the ratio of geometric and arithmetic means of the eigenvalues of $\mathbf{H H}^{H}$. $S_{\mathrm{ED}}$ is a useful figure of merit to characterize ED by a single number [15]. It is noted that in the case of small ED, $S_{\mathrm{ED}}$ tends to unity $\left(S_{\mathrm{ED}} \rightarrow 1\right)$, whereas, in the case of high ED, $S_{\mathrm{ED}}$ tends to zero $\left(S_{\mathrm{ED}} \rightarrow 1\right)$.

Table 4 shows the mean value and standard deviation of the eigenvalue dispersion of MIMO channel for the field CIRs $\mathbf{H}^{\text {meas }}$ and the reconstructed CIRs $\mathbf{H}^{\text {model }}$. As shown in Table 4, for all the four cases, the mean values are, respectively, $0.65,0.77,0.82$, and 0.82 . This means that the reconstructed data has a larger $S_{\mathrm{ED}}$ than the real data, so the modeling method underestimates the spatial correlation of MIMO channel. And when we take the standard deviation into account, a higher value will cause a significant change in spatial correlation. Comparing the $\mathbf{H}^{\text {meas }}$ with the CIRs $\mathbf{H}^{\text {model }}$, the reconstructed data has a larger standard deviation than the field measurement data.

Because of the coupling among the antennas in real environment, the correlation of practical antenna array will increase. However, the IMT-Advanced channel model does not take this nonideal issue into account. This antenna modeling method will make the spatial fading of the parallel channels more independently than real measurement. As the results shown above, using the data from channel reconstruction will underrate the spatial correlation in MIMO system and impose an impact on the spatial domain. In order to obtain the expected multiplexing degree from real MIMO system, the spatial resource (such as antenna spacing and polarized antenna) must be more utilized than that used in channel modeling.

3.3. Channel Capacity. The channel capacity is one of the important indicators for MIMO channel. In the absence of the channel state information at the transmitter, it is optimal to equally allocate power over all antennas. The channel capacity of frequency-selective fading MIMO channel is given by [16]

$$
C(t)=\frac{1}{B} \int_{B} \log _{2} \operatorname{det}\left(\mathbf{I}_{U}+\frac{\rho}{\beta S} \mathbf{H}(t, f) \mathbf{H}^{H}(t, f)\right) d f,
$$

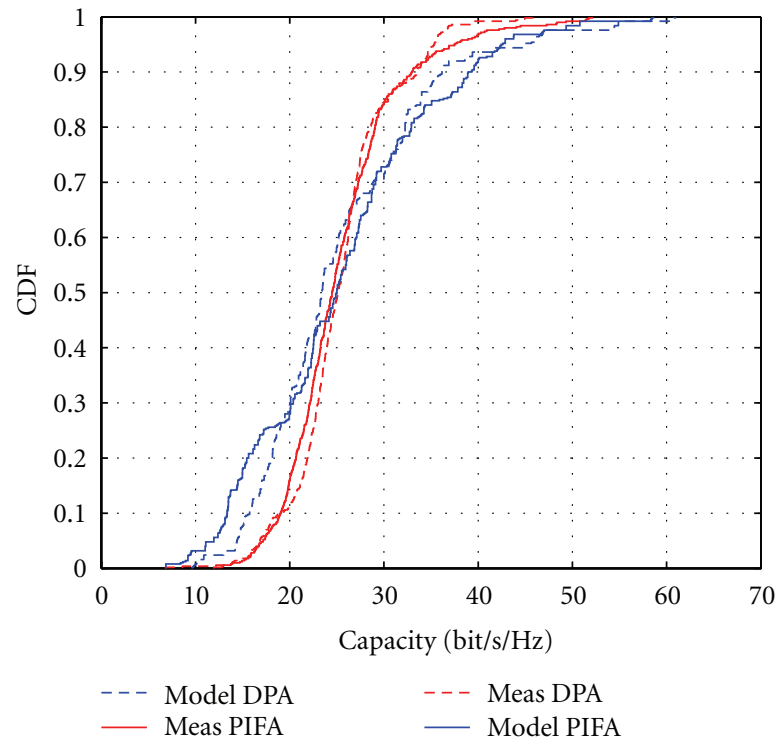

FIgURE 6: Capacity CDF at SNR $25 \mathrm{~dB}$.

where $\rho$ denotes the SNR and $B$ is the bandwidth. For the discrete channel $H(j, k)$, an approximation can be given by

$$
C(j) \approx \frac{1}{K} \sum_{k=1}^{K} \log _{2} \operatorname{det}\left(\mathbf{H}_{U}+\frac{\rho}{\beta S} \mathbf{H}(j, k) \mathbf{H}^{H}(j, k)\right),
$$

where $K$ is the number of frequency bins of $j$ th time realization and $\beta$ is a common normalization factor for all channel realizations in such that the average channel power gain is unitary as

$$
E\left\{\frac{1}{\beta}\|\mathbf{H}(j, k)\|_{F}^{2}\right\}=U \cdot S,
$$

where $\|\cdot\|_{F}$ denotes the Frobenius norm. The CIRs between different Tx-Rx antenna array pairs are used to form the channel transfer matrix. After transforming the transfer matrix to the frequency domain, we can calculate the capacity using (14). Figure 6 shows the capacity cumulative density function (CDF) curves for all types of channel realizations.

As is shown in Figure 6, for both the field CIRs, the $\mathbf{H}_{\mathrm{PIFA}}^{\text {meas }}$ has the same outage capacity as the $\mathbf{H}_{\mathrm{DPA}}^{\text {meas }}$ and for the reconstructed CIRs, the similar result is presented. But comparing the field CIRs with the reconstructed CIRs, it is should be noted that the outage capacity $\mathrm{CDF}$ of $\mathbf{H}_{\mathrm{DPA}}^{\mathrm{meas}}$ has a smaller slope than that of $\mathbf{H}_{\mathrm{DPA}}^{\text {model }}$. This is the reason why the field measurement data has a larger 5\% channel outage capacity than the reconstructed channel data. As shown in Figure 7 , the $5 \%$ channel outage capacity value of $\mathbf{H}_{\mathrm{DPA}}^{\mathrm{meas}}$, $\mathbf{H}_{\mathrm{DPA}}^{\mathrm{model}}, \mathbf{H}_{\mathrm{PIFA}}^{\mathrm{meas}}$, and $\mathbf{H}_{\mathrm{PIFA}}^{\mathrm{model}}$ are, respectively, $16.86,14.62,17.1$, and 12.38. The reason is the incorrect estimation in the standard deviation of the eigenvalue dispersion. Because the standard deviation of the eigenvalue dispersion from the reconstructed CIRs $\mathbf{H}^{\text {model }}$ is larger than that from the field CIRs $\mathbf{H}^{\text {meas }}$, the reconstructed data has a larger slope and a higher $5 \%$ outage capacity. 


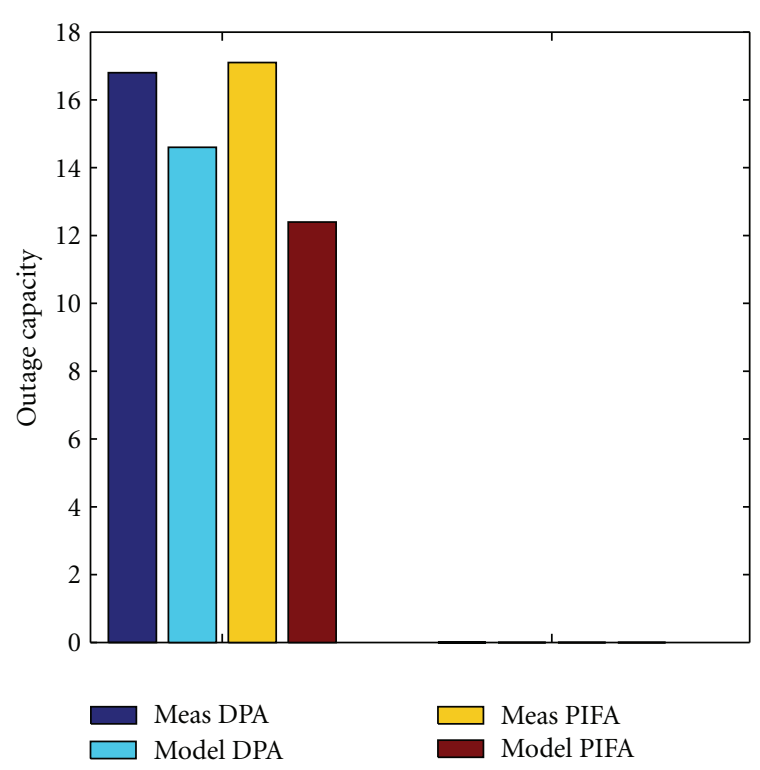

FIGURE 7: 5\% outage channel capacity at SNR $25 \mathrm{~dB}$ at the different spots.

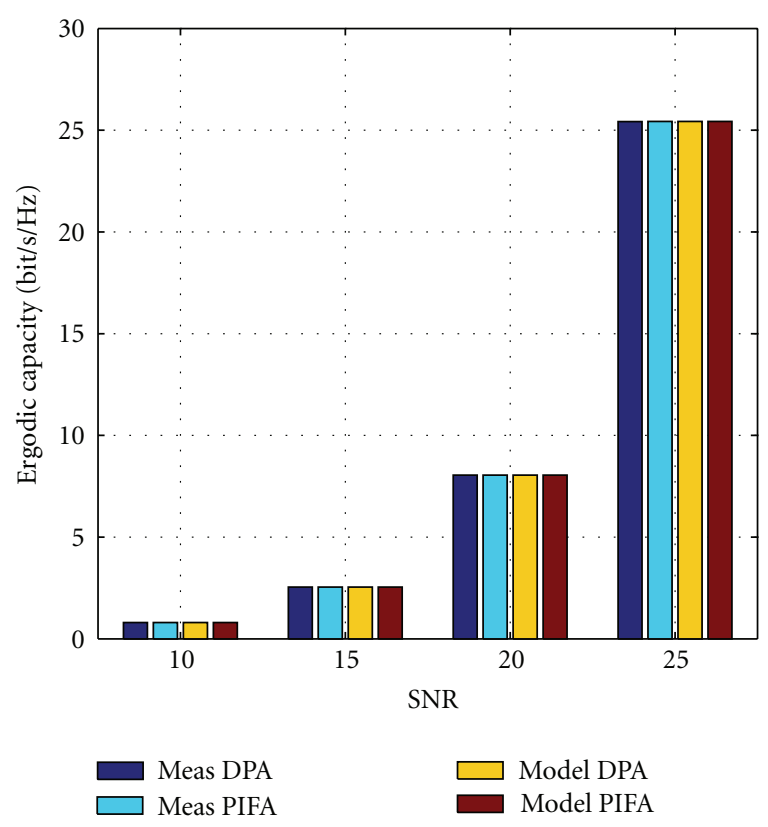

FIgURE 8: Ergodic capacity.

On the other hand, under the condition of block fading channel, the ergodic capacity is used to characterize the spatial channel capacity. Figure 8 show the ergodic capacity of MIMO channel for all four cases at spot 4. It is obvious that at each SNR all the cases have a similar ergodic capacity. Because both the $\mathbf{H}^{\text {meas }}$ and $\mathbf{H}^{\text {model }}$ have a large $S_{\mathrm{ED}}$, the spatial correlation of them is small enough to make them have a high ergodic capacity.

\section{Conclusion}

In this paper, the antenna pattern modeling of IMTAdvanced channel model is validated by some extensive measurement campaign at center frequency of $2.35 \mathrm{GHz}$ with $50 \mathrm{MHz}$ effective bandwidth in indoor environment. DPA, PIFA, and ODA are used to collect the spatial CIRs, and the channel reconstruction is performed by updating the characteristics parameters of IMT-Advanced channel model and matrix manipulation with the antenna array patterns at the both sides of the communication link. The coherent bandwidth, eigenvalue dispersion, and channel capacity are compared between the measured raw data and the reconstructed CIRs. It is found that the reconstructed data can well fit to the field measurement data in delay domain. Meanwhile, different from CIRs with DPA, the field CIRs with PIFA have a smaller coherent bandwidth than the reconstructed CIRs. A larger standard deviation of the eigenvalue dispersion makes the reconstructed CIRs have a wider range on spatial correlation than the measured raw data. So the IMT-Advanced channel model will have an influence on the channel spatial correlation estimation. On the channel capacity prediction, the wider range on spatial correlation causes the underestimation of the $5 \%$ channel outage capacity, but the four cases all have a consistent ergodic capacity at each SNR. So it is concluded that the modeling method based on IMT-Advanced channel model has a larger impact on the spatial characteristics than the frequency characteristics. Because of the nonideal issues on the antennas, the incorporation of the antenna array on the channel model should be further considered for future channel measurement and modeling.

\section{Acknowledgments}

The research is supported in part by National Natural Science Foundation of China with NO. 61171105, National Key Technology Research and Development Program of the Ministry of Science and Technology of China with NO. 2012BAF14B01, and Program for New Century Excellent Talents in University of Ministry of Education of China with NCET-11-0598, as well as funded by China Academy of Telecommunications Technology.

\section{References}

[1] G. J. Foschini and M. J. Gans, "On limits of wireless communications in a fading environment when using multiple antennas," Wireless Personal Communications, vol. 6, no. 3, pp. 311-335, 1998.

[2] E. Telatar, "Capacity of multi-antenna gaussian channels," European Transactions on Telecommunications, vol. 10, no. 6, pp. 585-595, 1999.

[3] D.-S. Shiu, G. J. Foschini, M. J. Gans, and J. M. Kahn, "Fading correlation and its effect on the capacity of multielement antenna systems," IEEE Transactions on Communications, vol. 48, no. 3, pp. 502-513, 2000.

[4] J. Zhang, "Review of wideband MIMO channel measurement and modeling for IMT-advanced systems," Chinese Science Bulletin, vol. 57, no. 19, pp. 2387-2400, 2012. 
[5] D. Chizhik, F. Rashid-Farrokhi, J. Ling, and A. Lozano, "Effect of antenna separation on the capacity of BLAST in correlated channels," IEEE Communications Letters, vol. 4, no. 11, pp. 337-339, 2000.

[6] K. Yu, M. Bengtsson, B. Ottersten, D. McNamara, P. Karlsson, and M. Beach, "A wideband statistical model for NLOS indoor MIMO channels," in Proceedings of the IEEE 55th Vehicular Technology Conference (VTC '02), vol. 1, pp. 370-374, May 2002.

[7] H. Özcelik, M. Herdin, W. Weichselberger, J. Wallace, and E. Bonek, "Deficiencies of "Kronecker" MIMO radio channel model," Electronics Letters, vol. 39, no. 16, pp. 1209-1210, 2003.

[8] A. M. Shirook, M. Ali, and P. Lusina, "User effects on MIMO performance: from an antenna to a link perspective," International Journal of Antennas and Propagation, vol. 2011, Article ID 918315, 13 pages, 2011.

[9] FEKO User Manual, Suite 5.4, EM Software \& Systems-S.A. (Pty), 2008.

[10] Y. Zhang, J. Zhang, G. Liu, X. Gao, and P. Zhang, "A generic validation framework for wideband MIMO channel models," in Proceedings of the IEEE Vehicular Technology Conference (VTC'08), pp. 330-334, May 2008.

[11] Guidelines for Evaluation of Radio Interface Technologies for IMTAdvanced, ITU-R Std, 2008.

[12] M. Steinbauer, A. F. Molisch, and E. Bonek, "The doubledirectional radio channel," IEEE Antennas and Propagation Magazine, vol. 43, no. 4, pp. 51-63, 2001.

[13] Propsound cs: The Ultimate Technology in Radio Propagation Measurement, Elektrobit.

[14] X. Nie, J. H. Zhang, C. Huang, Z. M. Liu, and P. Zhang, "Spatial characteristics and capacity investigation of indoor hotspot channel based on wideband MIMO measurement at 4.9 GHz," Journal of China Universities of Posts and Telecommunications, vol. 17, no. 3, pp. 38-44, 2010.

[15] P. Suvikunnas, J. Salo, L. Vuokko, J. Kivinen, K. Sulonen, and P. Vainikainen, "Comparison of MIMO antenna configurations: methods and experimental results," IEEE Transactions on Vehicular Technology, vol. 57, no. 2, pp. 1021-1031, 2008.

[16] A. Paulraj, R. Nabar, and D. Gor, Introduction to Space-Time Wireless Communications, Cambridge University Press, 2003. 

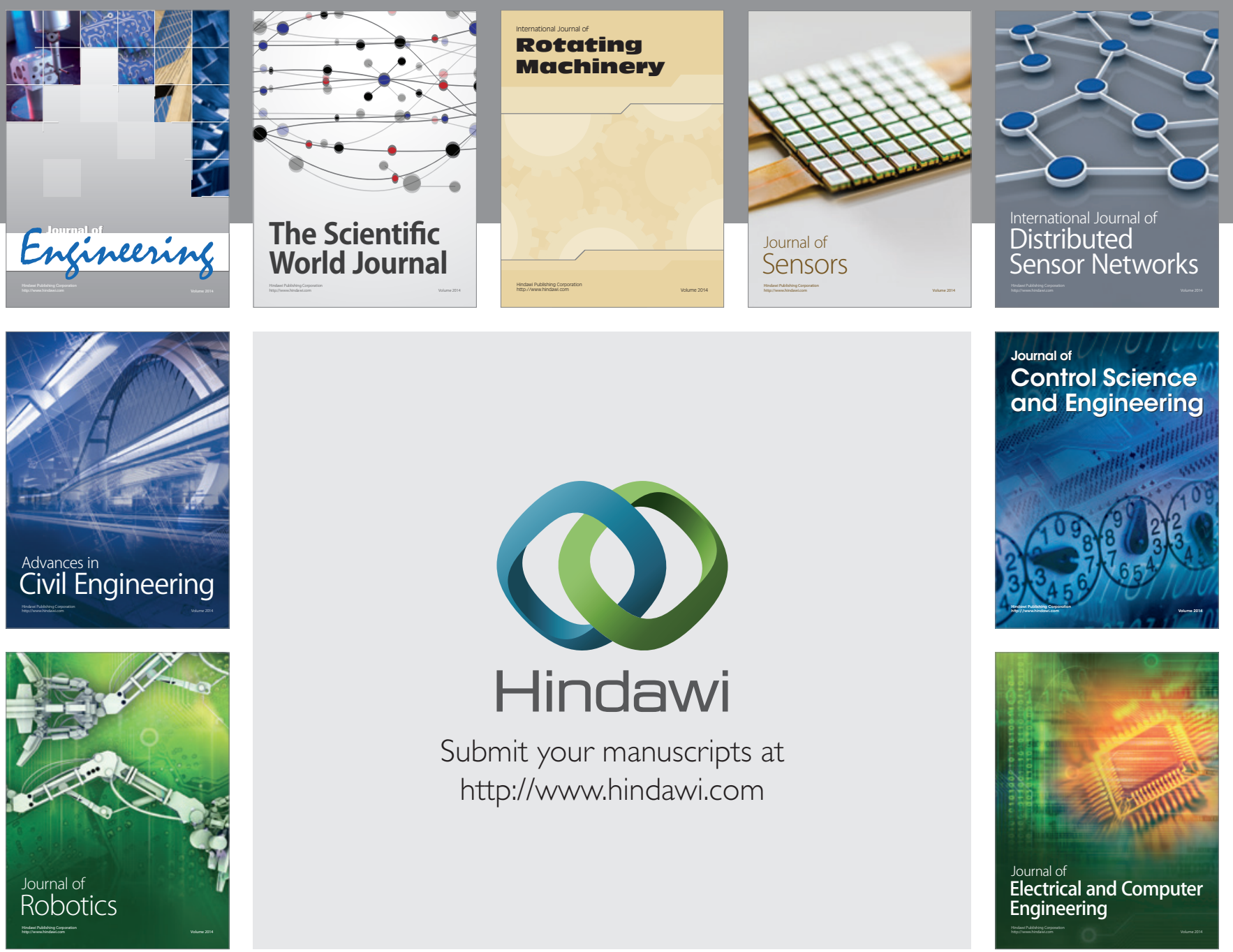

Submit your manuscripts at

http://www.hindawi.com
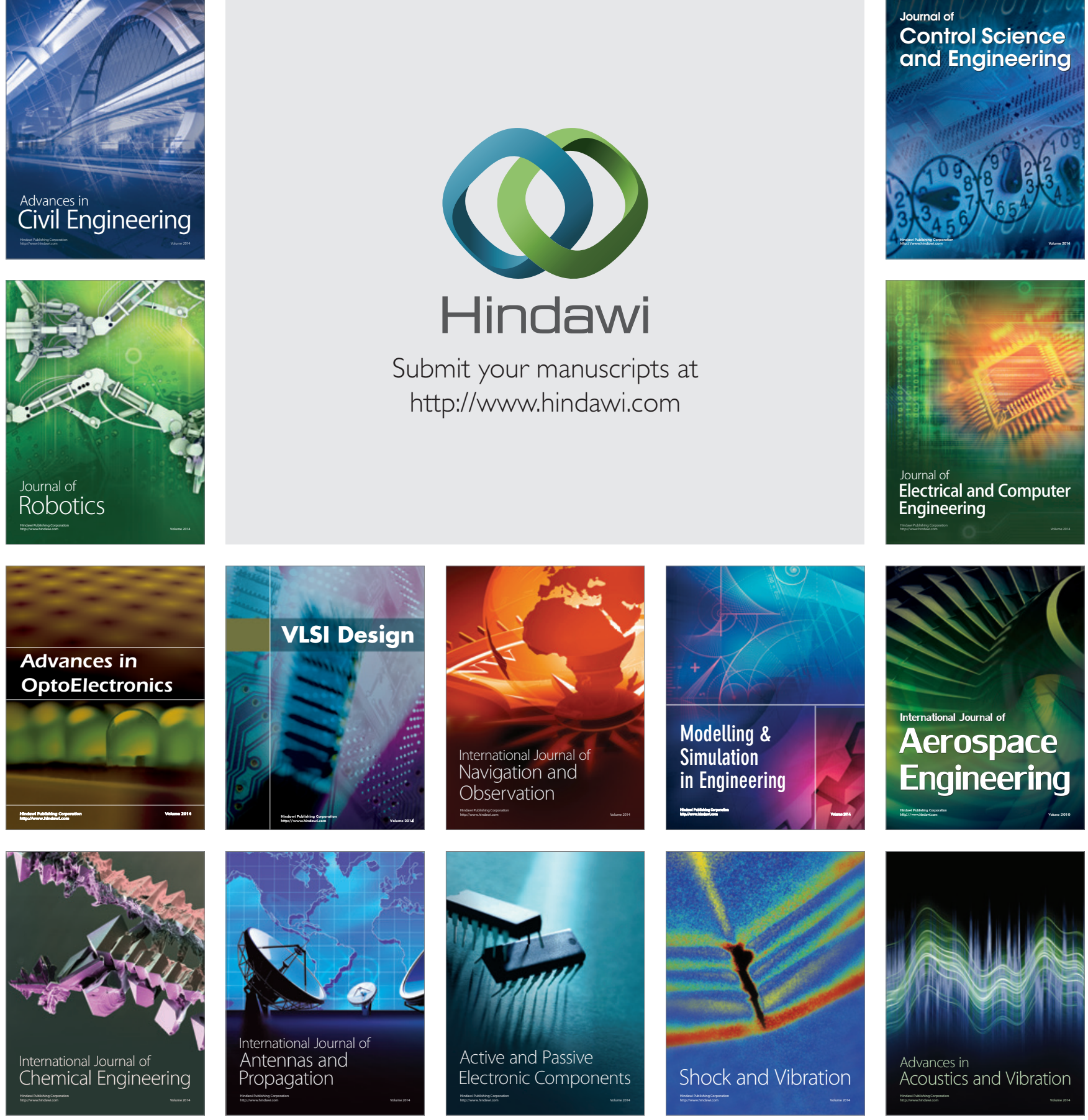